\title{
Révolution(s) du roman, de Blanche de Beaulieu (1826) à Création et Rédemption (1869)
}

\author{
Julie Anselmini \\ Université de Caen Normandie \\ julie.anselmini@aliceadsl.fr
}

Rebut: 4 de febrer de 2021

Acceptat: 1 de març de 2021

\begin{abstract}
RESUM
Revolucio(ns) de la novela, de Blanche de Beaulieu (1826) à Création et Rédemption (1869)

Els inicis de la carrera de Dumas com a escriptor de ficció narrativa guarden una curiosa semblança amb el final de la seva carrera: tant el relat curt Blanche de Beaulieu (1826) com la novel-la Création et Rédemption (1869) estan ambientats durant el Terror i tracten de la relació amorosa frustrada entre una jove aristòcrata i un republicà. L'article compara aquests dos extrems de la carrera de Dumas per tal de comprendre l'evolució essencial que marca l'obra de Dumas, tant en l'escriptura de ficció històrica com en la representació de la Revolució i el Terror.
\end{abstract}

Paraules clau

Dumas pare, ficció narrativa, Història, Revolució, Terror.

RÉSUMÉ

Révolution(s) du roman, de Blanche de Beaulieu (1826) à Création et Rédemption (1869)

Les tout débuts de Dumas en tant qu'auteur de fictions narratives présentent d'étranges similitudes avec la toute fin de sa carrière : dans la nouvelle Blanche de Beaulieu (1826) comme dans le roman Création et Rédemption (1869), l'action se situe à l'époque de la Terreur et gravite autour des amours contrariées d'une jeune aristocrate et d'un républicain. L'article confronte ces deux extrémités de la carrière de Dumas pour, à la lumière de cette confrontation, saisir les évolutions essentielles qui marquent l'œuvre dumasienne, à la fois 
en ce qui concerne l'écriture de la fiction historique et la représentation de la Révolution et de la Terreur.

MotS Clés

Dumas père, fiction narrative, Histoire, Révolution, Terreur.

RESUMEN

Revolucion(es) de la novela, de Blanche de Beaulieu (1826) à Création et Rédemption (1869)

Los inicios de la carrera de Dumas como escritor de ficción narrativa guardan un extraño parecido con el final de su carrera: tanto el relato corto Blanche de Beaulieu (1826) como la novela Création et Rédemption (1869) están ambientados durante el Terror y giran en torno a la relación amorosa frustrada entre una joven aristócrata y un republicano. El artículo compara estos dos extremos de la carrera de Dumas para comprender la evolución esencial que marca la obra de Dumas, tanto en la escritura de ficción histórica como en la representación de la Revolución y el Terror.

Palabras Clave

Dumas padre, ficción narrativa, Historia, Revolución, Terror.

Abstract

Revolution(s) of the novel, from Blanche de Beaulieu (1826) to Création et Rédemption (1869)

The very beginnings of Dumas as an author of narrative fiction show strange similarities with the very end of his career: in the short story Blanche de Beaulieu (1826) as in the novel Création et Rédemption (1869), the action unfolds during the Reign of Terror and revolves around two star-crossed lovers, a young aristocrat and a Republican. This article examines both ends of Dumas' career and, in light of this examination, aims to foreground the essential evolutions that characterize the work of Dumas, in relation to both the writing of historical fiction and the representation of the Revolution and the Reign of Terror.

KEYWORDS

Dumas père, narrative fiction, History, Revolution, Reign of Terror. 
Partons des tout débuts de la carrière de Dumas comme auteur de fictions narratives : en 1826, il fait paraître chez l'éditeur Sanson trois

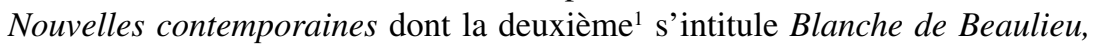
ou la Vendéenne. L'action de cette nouvelle se passe en 1793 : en pleine guerre civile, Olivier et d'Hervilly, deux généraux républicains, sont envoyés dans le bocage vendéen ; ils y assistent à un carnage de royalistes par des républicains, au terme duquel un jeune Vendéen vient supplier Olivier de le sauver, avant de perdre connaissance. Dégrafant son habit, Olivier découvre qu'il s'agit d'une jeune fille. Olivier et d'Hervilly la prennent sous leur protection et Olivier demande une permission pour se rendre à Nantes, où réside sa famille, afin de mettre sa protégée en sécurité. Mais le représentant du peuple Delmar surprend Blanche et la fait arrêter, alors qu'Olivier a reçu l'ordre de rejoindre l'armée. Celui-ci retourne à Nantes et force les portes de la prison. Là, il demande à Blanche de devenir sa femme, persuadé que Carrier ne fera pas exécuter l'épouse d'un général républicain. Les jeunes gens reçoivent la bénédiction d'un prêtre réfractaire et Olivier part à bride abattue vers Paris, où Robespierre accepte de gracier Blanche. Mais, malgré sa célérité pour revenir à Nantes, Olivier arrive au moment où le bourreau brandit la tête de Blanche décapitée pour la montrer à la foule...

Cette nouvelle reparait en juillet 1831 dans une version modifiée et sous le titre de La Rose rouge dans La Revue des Deux Mondes. La principale modification tient au remplacement des personnages fictifs Olivier et d'Hervilly par deux personnages historiques : respectivement par le général Marceau, et par le propre père de Dumas, le général Dumas, qui s'est en effet trouvé en Vendée quelques jours en décembre 1793 en tant que commandant en chef de l'armée des Pyrénées occidentales, alors qu'il conduisait un renfort à l'armée de l'Ouest. C'est sous cette nouvelle version que la nouvelle, à nouveau sous le titre de Blanche de Beaulieu, sera recueillie en 1835 dans les Souvenirs d'Antony paru chez Louis Dumont (et c'est sur cette version de 1831 que seront fondées les analyses suivantes, pour des raisons éditoriales ${ }^{2}$ ).

Faisons à présent un saut jusqu'à l'autre extrémité de la carrière de Dumas en tant qu'auteur de fictions narratives : de décembre 1869 à mai 1870, il fait paraître dans les colonnes du journal Le Siècle son dernier grand roman achevé, Création et Rédemption, qui ne sortira en librairie qu'à titre posthume, en 1872, chez Michel Lévy, sous la forme de deux volumes intitulés

\footnotetext{
${ }^{1}$ Les autres s'intitulent Laurette et Marie.

${ }^{2}$ Notre édition : Alexandre Dumas, Blanche de Beaulieu, texte établi et présenté par J. Thibaudeau, Mercure de France, Paris, « Le petit Mercure », 1996.
} 
Le Docteur mystérieux et La Fille du marquis. Il est impossible de revenir en détail sur l'intrigue de ce long roman foisonnant, intrigue que je serai amenée à évoquer plus loin. Ce qu'il importe de souligner, c'est que l'action de ce roman tardif, située entre 1785 et 1796, présente d'étranges similitudes avec l'action de la nouvelle des débuts, Blanche de Beaulieu : une jeune aristocrate, Éva, aime un républicain, Jacques, qui lui a sauvé la vie, et elle se voit plongée en 1793 dans la tourmente de la Terreur, période au cours de laquelle elle manque être décapitée et réchappe de justesse à la mort.

Dumas a consacré bien d'autres œuvres à la période de la Révolution et de la Terreur, qui l'intéresse entre toutes, car elle est à ses yeux, comme aux yeux d'autres écrivains et penseurs du XIX ${ }^{\mathrm{e}}$ siècle, l'époque climatérique qui a fait basculer la France dans la modernité, mais aussi l'épisode traumatique que tout le siècle cherche à digérer, à surmonter, pour pouvoir envisager résolument l'avenir ${ }^{3}$. Parmi les œuvres de Dumas consacrées à la Révolution, on pourrait citer, parus entre 1846 et 1867, Le Chevalier de Maison-Rouge (1846), Joseph Balsamo (1848), Le Collier de la reine (1850), Ange Pitou (1851), La Comtesse de Charny (1855), Ingénue (1854), René Besson (1862) ou encore Les Blancs et les Bleus (1867)... Sans m'attarder sur ces jalons intermédiaires, tout importants qu'ils soient (notamment Mémoires d'un médecin ${ }^{4}$ et Le Chevalier de Maison-Rouge), je voudrais, de façon assez libre, prendre ces deux extrémités de la carrière de Dumas, Blanche de Beaulieu et Création et Rédemption, pour les confronter. À la lumière de cette confrontation, il s'agira de saisir, comme dans un miroir grossissant, les évolutions essentielles qui marquent l'œuvre dumasienne, à la fois en ce qui concerne l'écriture de la fiction historique et la représentation de la Révolution et de la Terreur.

\section{$* * *$}

Le contraste entre les deux œuvres tient d'abord à la différence de genres qui les distingue : Blanche de Beaulieu est une nouvelle, un récit bref, concentré, tendu vers sa chute, tandis que Création et Rédemption est un vaste roman de plusieurs centaines de pages. L'action est beaucoup plus resserrée

\footnotetext{
${ }^{3}$ Sur cette question, je me permets de renvoyer à mon article : «Franchir le fleuve de sang : représentation et transgression dans les romans de la Terreur d'Alexandre Dumas », in Les Frontières en question, sous la dir. de N. Auzas, N. Cohen et S. Scarpa, Presses universitaires de Grenoble, 2007, pp. 189-199.

${ }^{4}$ Tétralogie comprenant Joseph Balsamo, Le Collier de la reine, Ange Pitou et La Comtesse de Charny.
} 
dans la nouvelle, et correspond au temps, tragique, d'une crise tranchée par un événement fatal : à peine les deux jeunes gens, de camps opposés, sontils tombés amoureux, que Blanche est démasquée, arrêtée, emprisonnée, puis condamnée à mort malgré son mariage avec Marceau et la tentative de celuici pour la sauver. Le fait qu'il revient à Nantes cinq minutes trop tard pour sauver son épouse souligne bien qu'on est dans le temps de l'urgence, de la précipitation, dans un temps qui manque, qui fait défaut.

Le roman, lui, décrit de façon beaucoup plus ample et détaillée la naissance de l'amour entre les deux personnages, le savant docteur et républicain Jacques Mérey et la petite Éva, de son vrai nom Hélène de Chazelay, aristocrate abandonnée dans la cabane d'un braconnier par son noble père, le marquis de Chazelay, qui la juge frappée d'idiotisme. Le développement de cet amour correspond d'abord aux étapes du traitement et de l'éducation dont Jacques, qui a recueilli la fillette, la fait bénéficier, grâce à ses dons de magnétiseur. Il sauve et pétrit en quelque sorte à force de volonté et d'amour ce corps d'abord débile et cette âme, de sorte que le mythe de Pygmalion est à l'horizon de tout le roman. On en comprend mieux la première partie du titre, Création..., qui renvoie aussi au fantasme démiurgique qui habite Jacques : «C'était une nature de Dieu, incapable d'aimer un être qu'il n'aurait point créé lui-même ${ }^{5}$ ». Cet amour sera ensuite soumis à l'épreuve de la séparation, au cours des années révolutionnaires : Jacques, siégeant à la Convention, est envoyé en mission par Danton, à la frontière nord-est de la France puis à l'étranger; Éva, qui a été arrachée à son protecteur par le marquis de Chazelay, est exilée à Vienne avant de revenir à Paris, dans l'espoir de retrouver Jacques, en pleine Terreur, tandis que celui-ci est parti à Vienne pour essayer de la retrouver... Une fois cette époque sanglante refermée, les deux jeunes gens se retrouveront et auront encore à se réconcilier : Éva est en effet devenue la maîtresse de Barras au moment de Thermidor (été 1794), elle a donc commis une faute dont elle doit se racheter : ce qui explique la seconde partie du titre,... Rédemption.

La fin du roman, se concluant par le mariage d'Éva pardonnée et de Jacques, est optimiste et heureuse, tandis que le dénouement de la nouvelle était macabre et tragique. Le traitement de l'intrigue dans la nouvelle, au lieu de développer les tenants et les aboutissants de la période révolutionnaire et des différents soubresauts qui la scandent, se concentre en outre sur la guerre de Vendée, au sommet de la Terreur, et donne à voir, de façon obsédante, le sentiment aussi puissant que tétanisant qui a donné son nom à la période.

${ }^{5}$ Le Docteur mystérieux, Slatkine, Paris-Genève, « ressources », 1980, chap. XLIX, p. 22. 
Le récit de Blanche de Beaulieu, après quelques pages consacrées à une présentation du camp républicain et de ses protagonistes, s'ouvre sur une scène de carnage, qui provoque la peur panique du personnage éponyme, dont la frayeur dénonce le sexe : Marceau s'étonne en effet de cette émotion extrême chez ce jeune soldat vendéen, qui, évanoui, révèle qu'il est une jeune fille. En dépit de brèves périodes d'accalmie, le sentiment de terreur ne cesse de s'emparer de Blanche de Beaulieu, tout au long du récit. Confrontée au représentant du peuple Delmar, elle apparaît ainsi « debout, pâle et froide, comme une statue de la terreur ${ }^{6} »$; son agitation atteint un sommet d'intensité alors qu'elle a été jetée en prison, et que s'ensuivent des scènes frénétiques entre elle et Marceau, qui pense tomber fou à son tour devant la résistance de Blanche à l'épouser ${ }^{7}$... La dernière image que le lecteur, érigé en spectateur, aura de l'hérö̈ne éponyme, est celle de sa tête, une rose rouge serrée entre les dents, alors que le bourreau vient de la décapiter, tête coupée médusante qui est comme l'emblème de la Terreur... On en trouve de nombreuses chez Dumas ${ }^{8}$, mais celle-ci a ceci de particulièrement frappant qu'elle clôt brutalement la nouvelle :

En ce moment, le bourreau, saisissant par ses longs cheveux blonds la tête d'une jeune fille, présentait au peuple ce hideux spectacle ; la foule, épouvantée, se détournait avec effroi, car elle croyait lui voir vomir des flots de sangs !... Tout à coup, au milieu de cette foule muette, un cri de rage, dans lequel semblent s'être épuisées toutes les forces humaines, se fait entendre : Marceau venait de reconnaître, entre les dents de cette tête, la rose rouge qu'il avait donnée à la jeune Vendéenne9.

Blanche de Beaulieu cultive donc une esthétique frénétique et macabre encore très en vogue dans les années 1820 et 1830, et qu'on retrouvera dans les récits fantastiques que Dumas écrit en 1849, Les Mille et un Fantômes et La Femme au collier de velours, récits qui gravitent tous aussi, chacun à sa manière, autour du motif de la tête coupée et de la Terreur.

La chute de Blanche de Beaulieu est même si terrifiante qu'elle fait basculer la fin du texte dans un climat fantastique fondé sur un trouble violent,

\footnotetext{
${ }^{6}$ Blanche de Beaulieu, op. cit., p. 39.

${ }^{7}$ Voir Ibid., pp. 61-66.

${ }^{8}$ Même dans des récits non révolutionnaires : on songe notamment à la scène de décapitation de Milady dans Les Trois Mousquetaires.
}

${ }^{9}$ Blanche de Beaulieu, op. cit., p. 87. 
né de l'hésitation, chez le personnage et par répercussion chez le lecteur, entre le fait de croire à la réalité incroyable de ce qu'on voit, de regarder en face le spectacle traumatique, ou de l'interpréter comme le fruit d'un cauchemar, d'une hallucination ou d'une crise de folie : la fin de la nouvelle, focalisée sur le personnage de Marceau, adopte ainsi le point de vue et les réaction de celuici face à la vision bouleversante.

Dans Création et Rédemption, la terreur née de la « sanglante fantasmagorie ${ }^{10} \gg$ des événements de 1793 est également au centre d'une partie du roman : il s'agit de la partie durant laquelle Éva, livrée à elle-même, sans soutien, affronte à Paris le spectacle de la Terreur et assiste à des exécutions à la chaîne dont elle-même est sur le point d'être victime. Cette partie est racontée par Éva elle-même, sous la forme d'un journal intime que Jacques retrouve et dont il prend connaissance ${ }^{11}$, apprenant par cette lecture quelles épreuves Éva a dû affronter tandis qu'elle était séparée de lui... ce qui l'amènera à lui pardonner sa faute. Les événements de la Terreur sont donc directement racontés par Éva, à la première personne, soit à travers le point de vue et les émotions d'une jeune fille bouleversée, qui croit à plusieurs reprises perdre la raison, ou sombrer dans un cauchemar :

[...] je ne saurais moi-même me rendre compte de l'ébranlement étrange qui secoue mon cerveau. Je ne sais pas comment cela se fait, mais il me semble que je ne suis plus complètement maîtresse de moi-même, et qu'il y a en moi une fatalité plus forte que ma volonté [...].

J'ai parfois des espèces d'hallucinations pendant lesquelles il me semble que, le jour où j'ai pris place dans la charrette, j'ai été véritablement guillotinée. Je crois parfois en rêve que je sens la douleur de la hache passant entre les vertèbres de mon cou ; je me dis que depuis ce jour je suis morte et que c'est mon ombre qui croit vivre et s'agite encore sur la terre ${ }^{12}$. [...]

On retrouve donc dans le roman un climat fantastique, fondé sur le trouble, l'hésitation à opter pour une interprétation irrationnelle ou rationnelle des événements. Mais, dans le roman, le fantastique, le trouble, l'incompréhension, ne sont qu'une étape, qui sera en définitive surmontée au profit de l'apaisement et de la réconciliation, alors que dans la nouvelle initiale,

\footnotetext{
${ }^{10}$ Le Docteur mystérieux, op. cit., p. 266.

${ }^{11}$ « Le manuscrit » compose le très long chapitre IX de La Fille du marquis, chapitre lui-même divisé en vingt-neuf sections.

${ }^{12}$ La Fille du marquis, Nelson Éditeurs, Paris, 1962, p. 310.
} 
le fantastique clôt le récit sur un excès de trouble et de terreur qui laisse le personnage survivant plongé dans la stupéfaction, tout comme le lecteur.

Cette différence dans l'usage du fantastique et le statut de la terreur tient à une évolution majeure qui se lit entre les deux œuvres. C'est sur cette évolution d'ordre idéologique que je voudrais m'attarder à présent, après avoir souligné des différences d'ordres générique et esthétique.

Dumas, dès Blanche de Beaulieu et probablement avant cela, est républicain. On songe au choix fondateur qu'il fait, âgé d'une douzaine d'années seulement ${ }^{13}$, en adoptant le nom plébéien et glorieux de son père au détriment de celui, aristocratique, de son marquis de grand-père, Davy de la Pailleterie. Ses convictions républicaines s'affirmeront et s'exprimeront ensuite lors de divers épisodes : en juillet 1830, il participe activement aux Trois Glorieuses, puis, alors qu'il était le bibliothécaire adjoint du duc d'Orléans, il présente sa démission à Louis-Philippe au lendemain de l'accession de celuici au trône ; en 1848, il se présente comme candidat libéral aux élections à l'Assemblée constituante (tout en se démarquant clairement des socialistes et des « rouges »); après le 2 décembre 1851, il prend un temps le chemin de l'exil ${ }^{14}$, puis, pendant tout le Second Empire, il soutient dans ses romans et ses journaux le proscrit Hugo, son ami ; en 1860, enfin, il rejoint Garibaldi lors de l'expédition des Mille, en Italie, et fonde L'Indipendente pour soutenir sa cause.

Mais, au début des années 1830, Dumas, comme bien d'autres écrivains et penseurs de son temps, peine encore à envisager sereinement et à comprendre la période de la Terreur : « lequel de nous n'a pas eu toute son éducation politique à refaire avant d'oser envisager froidement ce chiffre [...] fatal $-93^{15}$ ? », demande-t-il. Pour Hugo, qui ne parviendra qu'au terme de sa carrière à écrire le roman sur la Révolution auquel il songe depuis longtemps, Quatrevingt-treize, la Terreur est ce « point noir dans le ciel bleu » de la Révolution : même pour des penseurs progressistes et républicains, tels que Hugo l'est devenu, la Terreur pose problème, en tant que paroxysme marqué

\footnotetext{
${ }^{13}$ Voir le chap. XXXI de Mes Mémoires.

${ }^{14}$ Pour des raisons il est vrai davantage financières et économiques (suite à la faillite du ThéâtreHistorique) que politiques.
}

${ }^{15}$ Blanche de Beaulieu, op. cit., p. 47. 
par la guerre civile entre républicains et royalistes et par l'exécution de dizaines de milliers d'ennemis, avérés ou présumés, de la Révolution ${ }^{16}$. Pour Dumas elle est également « ce fantôme sanglant qui [...] fit la Révolution si longtemps incomprise ${ }^{17} \gg$.

Dans Blanche de Beaulieu, on voit clairement combien ce spectre continue à fasciner et à épouvanter. Il y a bien une tentative, dans cette nouvelle, pour éclairer et justifier la Terreur : elle est placée dans la bouche de Robespierre. Alors que Marceau est venu lui réclamer la grâce de Blanche, le Montagnard demande au jeune général, malgré l'urgence de la situation, cinq minutes pour lui exposer sa vision de choses et se justifier des mesures extrêmes qu'il a dû prendre, mesures destinées selon lui à « régénérer » la nation ${ }^{18}$. Mais il est terriblement ironique que ces cinq minutes soient justement celles qui manquent à Marceau pour sauver Blanche, qu'il retrouve fraîchement décapitée... En outre, ce discours, directement placée dans la bouche de Robespierre, apparaît comme un morceau d'éloquence (prononcé au théâtre de l'Odéon, où se trouve Robespierre) et non comme une réflexion assumée par l'auteur-narrateur omniscient. Dans Blanche de Beaulieu, la Terreur n'est pas digérée, ni dépassée ; elle reste médusante, paralysant l'appréhension de l'avenir.

Qu'en est-il dans Création et Rédemption ? Selon moi, la pensée politique de Dumas y a atteint toute son ampleur, toute sa cohérence, et elle intègre désormais 93 à une vision globale et pacifiée de la période révolutionnaire. Dans le roman, se succèdent en effet trois différentes utopies (ou dystopies) qui permettent au romancier de formuler, c'est-à-dire de figurer, par les moyens propres de la fiction, sa pensée historique, politique et sociale ${ }^{19}$. Quels sont ces trois moments qui se succèdent dans le roman ? D'abord, une utopie apolitique, la petite utopie idyllique d'Argenton dans laquelle, coupés du monde, dans leur jardin paradisiaque, sont reclus Jacques et Éva. S'abat ensuite la grande tourmente révolutionnaire. Éva est arrachée à Jacques par le marquis de Chazelay et le privilège de sa caste se traduit pour elle par un

\footnotetext{
${ }^{16} \mathrm{Il}$ y eut 17000 personnes condamnées à mort par les tribunaux révolutionnaires, 23000 exécutées sans jugement, 150000 victimes civiles en Vendée, soit un quart de la population.

${ }^{17}$ Le Docteur mystérieux, op. cit., p. 247.

${ }^{18}$ Voir Blanche de Beaulieu, op. cit., pp. 83-85.

${ }^{19}$ Pour une analyse plus approfondie sur ce point, je me permets de renvoyer à mon article : " Écriture de la Révolution et Utopie chez A. Dumas père : Création et Rédemption » (communication au colloque international «L'utopie sociale dans la littérature française du XIX ${ }^{e}$ siècle », Université de Salerne, Italie, 5-6 décembre 2017), à paraître dans Francofonia, revue internationale de l'Université de Bologne, sous la dir. de B. Diaz et A. Silvestri, automne 2021.
} 
exil en Autriche, puis par le danger qu'elle encourt, en tant qu'aristocrate, lorsqu'elle revient à Paris. Quant à Jacques, il accomplit différentes missions dont le charge Danton au profit de la cause républicaine, mais il les accomplit essentiellement à l'étranger : il sert ainsi de guide au général Dumouriez dans la forêt d'Argonne lors de la campagne de Champagne, tout en soignant les blessés, et il rapporte à la Convention les drapeaux arrachés aux Autrichiens et aux Prussiens à Valmy, puis à Jemmapes (en septembre et novembre 92). Il passera ensuite la fin de la Révolution tout à fait à l'écart des événements, en Amérique, après la proscription des Girondins au printemps 93. C'est en témoin, plus qu'en acteur, qu'il assiste à la terrible mise en œuvre des idéaux républicains, marquée en 1793 et 1794 par l'élimination physique massive des ennemis de la Révolution - ce que Danton appelle «l'ouvrage noir ${ }^{20} »$ de la Révolution.

C'est de même en témoin hallucinée qu'Éva y assiste une fois revenue à Paris, en pleine Terreur, et son journal intime, retrouvé par Jacques, s'apparente à un récit de voyage en enfer :

Dans ces moments de visions sépulcrales, je te cherche partout [écrit-elle à Jacques]. Il me semble que nous ne sommes séparés que par des brouillards épais, dans lesquels nous errons tous les deux, et dans lesquels, en punition de quelque faute que je cherche à me rappeler en vain, nous sommes condamnés à errer continuellement sans nous retrouver jamais ${ }^{21}$.

Ce voyage (au cours duquel Éva ressent un sentiment de culpabilité dont elle cherche en vain la cause, mais qui renvoie manifestement à son ascendance aristocratique) retrace ainsi la découverte d'une véritable dystopie, un univers régi par des lois implacables et sanglantes, au profit d'un idéal dont le visage est pour le moment celui de la monstrueuse Méduse, et dont la silhouette est celle de la guillotine, comparée à un animal féroce dévorant sans répit ses milliers de proies...

Après Thermidor et la mise en place du Directoire, et une fois les jeunes gens réunis (en 1796), une dernière utopie viendra clore le roman : utopie à nouveau agreste, alors que Jacques et Éva, loin de la scène parisienne et politique, privilégient la philanthropie comme mode d'action et font régner la prospérité et l'harmonie à Argenton (dans le Berri), harmonie dont leur union sera finalement l'image. Toute réformiste et modérée soit-elle, cette dernière

\footnotetext{
${ }^{20}$ Le Docteur mystérieux, op. cit., p. 264.

${ }^{21}$ La Fille du marquis, op. cit., p. 310.
} 
utopie aboutit néanmoins à un dépassement des clivages politiques et sociaux : un homme du peuple, républicain, épouse une aristocrate, mariage qui offre la figure apaisée d'une France post-révolutionnaire réconciliée, où les fautes ont été expiées dans le sang et par conséquent pardonnées, et où s'exprime de la part de l'auteur « un républicanisme de cœur, quasiment utopique ${ }^{22} »$, qu'il $^{2}$ nomme encore sa « religion du monde nouveau ${ }^{23} »$.

Dans cette évolution de Dumas vers une vision apaisée et optimiste de la période révolutionnaire dans son ensemble et de ses suites, des influences extérieures auront compté : on pourrait notamment développer l'influence qu'a eue l'historien républicain Michelet et sa vaste Histoire de la Révolution sur notre auteur ${ }^{24}$, qui cite Michelet comme «mon maître, l'homme que j'admire comme historien, et je dirai presque comme poète au-dessus de tous ${ }^{25} »$. Mais Dumas, dans ce processus, aura aussi mis à profit tout son talent et sa maîtrise d'écrivain ${ }^{26}$, y compris des procédés développés en écrivant des nouvelles : l'usage du fantastique, notamment, lui permet de figurer du point de vue d'Éva, victime passive des événements, le traumatisme engendré par la Terreur, et de dépasser celui-ci une fois dissipé le trouble, une fois Éva sauvée. La figuration des acteurs de la Terreur tels que Danton ou Marat sous la forme de créatures mythologiques, titan sublime pour Danton ${ }^{27}$, créature monstrueuse pour Marat, sont un autre moyen pour exonérer le peuple français de ces crimes et pour

${ }^{22} \mathrm{~V}$. Frigerio, Les Fils de Monte-Cristo. Idéologie du héros de roman populaire, PULIM, Limoges, «Médiatextes », 2002, p. 19.

${ }^{23}$ Le Docteur mystérieux, op. cit., p. 262.

${ }^{24}$ Sur cette question, voir Isabelle Safa, «Éros et Clio. La représentation dumasienne de la Révolution au prisme de Michelet », in Dumas amoureux. Formes et imaginaires de l'éros dumasien, sous la dir. de J. Anselmini et C. Schopp, Presses universitaires de Caen, «Symposia », 2020, pp. 291-304.

${ }^{25}$ Le Docteur mystérieux, op. cit., p. 115.

${ }^{26}$ Sa maîtrise du roman historique permet à Dumas de figurer les contradictions de la période révolutionnaire et leur dépassement : les personnages historiques passent à l'arrière-plan, au profit de personnages fictifs de premier plan qui peuvent être des témoins plus détachés de celle-ci, et qui bénéficient surtout d'une plus grande liberté d'action, puisqu'ils ne sont pas prisonniers du donné historique.

${ }^{27}$ Voir sur ce point J. Anselmini, «Quand les pygmées devinrent géants : Alexandre Dumas et la Révolution française », in Les géants entre mythe et littérature, sous la dir. de M. Closson et M. White-Le Goff, Artois Presses Université, Arras, 2007, pp. 171-179. 
figurer les mystères de ce bouleversement historique, mis en œuvre par des êtres hors de l'humanité, pourtant suscités et guidés par la Providence dans l'accomplissement de leurs «missions terribles ${ }^{28} »$.

Notons pour finir que le progrès dans la compréhension du devenir historique et de sa signification a aussi partie liée avec la complexité et l'autonomie plus grande accordée à la figure féminine. Blanche de Beaulieu est une jeune fille sans épaisseur, totalement passive et qui passe de la protection de son père à celle de Marceau, avant de finir, victime épouvantée, aux mains du bourreau. Si le personnage, au début, apparait déguisé en homme, c'est à des fins dramatiques et érotiques, mais jamais elle ne témoigne de qualités viriles ni d'une véritable volonté propre. Éva, elle, a beaucoup plus d'épaisseur et d'indépendance : d'abord victime d'abandon, faible et vulnérable, elle se fortifie et s'éduque. Si la nouvelle Blanche de Beaulieu montre la pétrification d'une jeune fille en statue, sous l'effet de la terreur, Création et Rédemption inverse cette fable et, conformément au mythe de Pygmalion, montre comment une statue inanimée devient jeune fille, s'animant et développant progressivement des forces physiques, émotionnelles, intellectuelles et spirituelles.

Outre le mythe de Pygmalion, c'est le mythe d'Orphée et Eurydice que récrit le roman. Pour Jacques il s'agit d'abord « de plonger au plus profond de cet enfer qu'on appelle l'imbécillité et de venir chercher une intelligence accroupie dans les ténèbres de la mort, et, comme Orphée avait fait pour Eurydice, la ramener malgré les dieux à la lumière du jour ${ }^{29} »$. Mais ces « ténèbres de la mort » seront une seconde fois traversées par Éva : c'est seule, livrée à ses propres ressources, qu'elle connaîtra les plus grandes épreuves de son histoire et de l'Histoire, se sauvant en perdant son intégrité et atteignant finalement le salut et le bonheur par-delà ces épreuves, par-delà ses propres fautes et sa culpabilité. Elle a, contrairement à la pâle Blanche, pleinement accédé au statut d'adulte ; elle s'est, autant que possible, émancipée, même si pour finir c'est la figure d'un couple qui triomphe, comme si chez Dumas la fondation d'une France républicaine se pensait sur le modèle d'une nouvelle alliance conclue entre un Adam et une Ėve rédimés du péché, mais par leur propre volonté.

$* * *$

${ }^{28}$ Le Docteur mystérieux, op. cit., p. 201 [deuxième partie].

${ }^{29}$ Le Docteur mystérieux, op. cit., p. 57. 
Pour conclure, on mesure à travers la confrontation de la nouvelle des débuts et du roman de la fin quelles constantes mais aussi quelles évolutions déterminantes marquent l'œuvre de Dumas. Ce que révèle de façon frappante cette confrontation, c'est que l'art du roman historique mis au point puis développé par Dumas est parfaitement adéquat à une pensée historique et politique. Dans Création et Rédemption, en effet, l'intrigue sentimentale et les aventures des personnages viennent réfléchir et symboliser le sens progressiste et la logique providentielle que Dumas assigne aux événements. On voit ainsi clairement pourquoi Dumas ne pouvait pas se cantonner dans le simple récit historique, lui qui charge la fiction romanesque, non seulement d'éclairer et de révéler le sens de l'Histoire, mais également d'en infléchir le cours, selon l'idéal démocratique qu'il forge de l'avenir. 